\title{
Magnetic Order and SdH Effect in Half-Heusler Phase ErPdBi
}

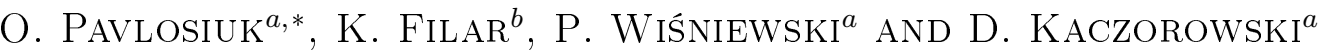 \\ ${ }^{a}$ Institute of Low Temperature and Structure Research, Polish Academy of Sciences, \\ P. Nr. 1410, 50-950 Wrocław, Poland \\ ${ }^{b}$ International Laboratory of High Magnetic Fields and Low Temperatures, Gajowicka 95, 53-421 Wrocław, Poland
}

\begin{abstract}
Single crystals of ErPdBi were grown from Bi-flux. The crystal structure of MgAgAs-type was confirmed using X-ray diffraction. Magnetization, magnetic susceptibility, electrical resistance and heat capacity measurements revealed an antiferromagnetic phase transition at $T_{\mathrm{N}}=1.2 \mathrm{~K}$. At high temperatures, the electrical resistance has a semiconducting-like character $(\mathrm{d} R / \mathrm{d} T<0)$. The resistance starts decreasing with decreasing $T$ below $15 \mathrm{~K}$ and shows a very sharp drop below $T_{\mathrm{N}}$ but remains finite down to $0.4 \mathrm{~K}$. Hence, no obvious evidence of superconductivity was found in the electrical transport data. On the other hand, the real part of AC magnetic susceptibility is negative below $T_{\mathrm{C}}=1.6 \mathrm{~K}$ and its imaginary component has a clear maximum at this temperature that might be associated with the onset of superconducting state. The electrical resistance revealed Shubnikov-de Haas oscillations in magnetic fields 8-33 T. Their amplitude decreases with increasing $T$ and disappears above $10 \mathrm{~K}$. Cyclotron mass determined from this dependence is $0.21 \mathrm{~m}_{\mathrm{e}}$.
\end{abstract}

DOI: 10.12693/APhysPolA.127.656

PACS: 74.10.+v, 75.40.Cx, 71.18.+y, 71.20.Eh, 72.15.Gd

\section{Introduction}

An equiatomic compound ErPdBi crystallizes in a noncentrosymmetric structure of the half-Heusler MgAgAstype (space group $F \overline{4} 3 m$ ) [1]. Similar rare-earth $(R E)$ based $R E \mathrm{PdBi}$ phases [2-4] exhibit localized antiferromagnetic behavior below the ordering temperature $T_{\mathrm{N}} \sim 2-13 \mathrm{~K}$. It has recently been proposed that some of these compounds can be considered as topological insulators (TI) $[5,6]$, i.e. analogs of the intensively studied $\mathrm{Hg}(\mathrm{Cd}) \mathrm{Te}$ and $\mathrm{Bi}_{2} \mathrm{Se}_{3}$ systems. TI is a novel state of quantum matter having a full insulating gap in the bulk and topologically protected gapless surface or edge states on the boundary [7]. The family of half-Heusler compounds includes not only metallic and semiconducting materials, but also superconducting compounds which exhibit band inversion. Such unique combination of superconductivity and band inversion, seen e.g. in LaPtBi [8], YPtBi $[9,10]$ and LuPtBi [11] makes these ternaries promising candidates for topological superconductors. Furthermore, a coexistence of superconductivity $\left(T_{\mathrm{c}}=1.22 \mathrm{~K}\right)$ and antiferromagnetic order $\left(T_{\mathrm{N}}=1.06 \mathrm{~K}\right)$ has recently been reported for a potentially topological compound ErPdBi [12]. However, as stated by authors of Ref. 12, their single crystals may have been contaminated by an impurity phase $\mathrm{PdBi}_{2}$ superconducting below $1.7 \mathrm{~K}$, and thus strongly influenced the observed physical properties of the samples studied.

\section{Experimental details}

High quality single crystals of $\mathrm{ErPdBi}$ were grown in $\mathrm{Bi}$ flux. The X-ray diffraction analysis revealed single phase with the MgAgAs-type cubic crystal structure and the

*corresponding author; e-mail: o.pavlosiuk@int.pan.wroc.pl lattice parameter of $6.589 \AA$, consistent with the previous reports $[1-4,12]$. Electrical transport and heat capacity measurements were performed in the temperature range from $0.4 \mathrm{~K}$ to $300 \mathrm{~K}$ using a Quantum Design Physical Property Measurement System (PPMS). Electrical resistance measurements in high magnetic fields up to $34 \mathrm{~T}$ were performed in a $10 \mathrm{~ms}$ pulse magnet. $\mathrm{DC}$ and $\mathrm{AC}$ magnetic susceptibility measurements were carried out in the temperature interval $0.5-1.8 \mathrm{~K}$ using a Quantum Design MPMS-XL magnetometer equipped with an iHelium3 refrigerator.

\section{Results and discussion}

The low-temperature dependence of the magnetic susceptibility of ErPdBi is shown in Fig. 1. Magnetic susceptibility was measured in zero-field-cooled (ZFC) and field-cooled (FC) regimes. Maxima in both $\chi(T)$ curves indicate antiferromagnetic state below $T_{\mathrm{N}}=1.2 \mathrm{~K}$. The field variation of the magnetization (inset of Fig. 1) shows no hysteresis nor remanence, and linear $\sigma(B)$ is typical for an antiferromagnet (abrupt change of slope at $2 \mathrm{~T}$ reflects a metamagnetic transition). The $\chi_{\mathrm{ZFC}}(T)$ and $\chi_{\mathrm{FC}}(T)$ curves split below the temperature of $1.65 \mathrm{~K}$, which coincides with features in the temperature dependences of the $\mathrm{AC}$ magnetic susceptibility.

As can be inferred from Fig. 2, the real component $\chi^{\prime}$ of the AC susceptibility is negative below $\sim 1.6 \mathrm{~K}$ and saturates at a small positive value above $1.7 \mathrm{~K}$. The imaginary component $\chi^{\prime \prime}$ has a maximum at $1.6 \mathrm{~K}$, which does not move with changing the frequency of driving field. Following Ref. [12], these features can be associated with the onset of superconducting state in $\mathrm{PdBi}_{2}$ that is likely present in the single crystals studied here as a minor phase.

The low-temperature specific heat of ErPdBi (Fig. 3) exhibits a clear lambda-shaped anomaly at $T_{\mathrm{N}}=$ $1.2 \mathrm{~K}$ that manifests the antiferromagnetic phase transition. In applied magnetic field the peak in $C(T)$ broadens 


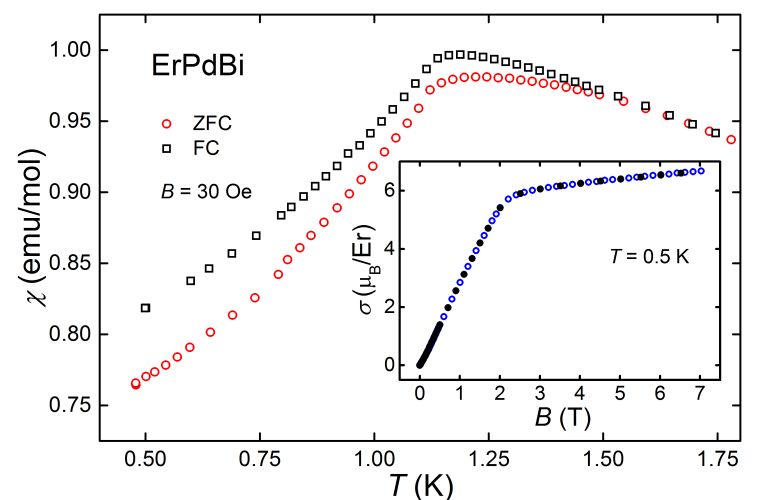

Fig. 1. DC magnetic susceptibility versus temperature measured in $\mathrm{ZFC}$ and $\mathrm{FC}$ regimes in a field of $30 \mathrm{Oe}$. Inset: Magnetic field variation of the magnetization taken at $0.5 \mathrm{~K}$ with increasing (empty symbols) and decreasing (solid symbols) magnetic field.

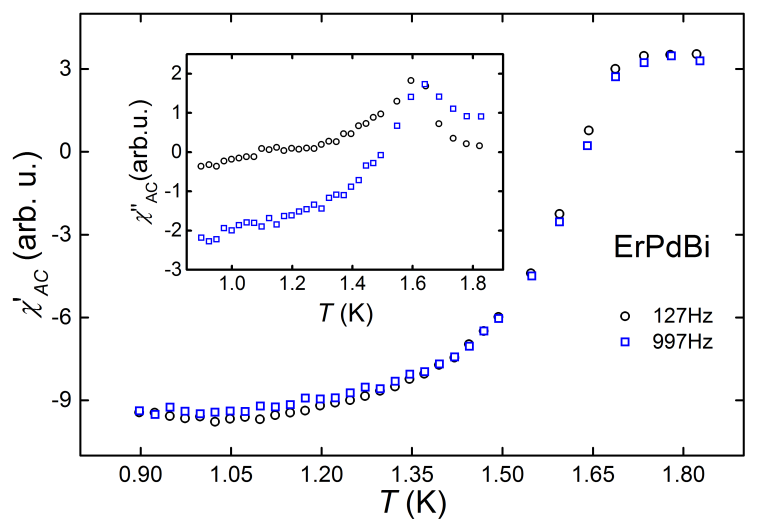

Fig. 2. Temperature dependence of the real and imaginary (inset) AC magnetic susceptibility measured in zero DC field with a 0.027 Oe driving field of different frequency.

and shifts to lower temperatures, as expected for antiferromagnets.

Figure 4 displays the temperature dependence of the electrical resistance of ErPdBi. Above about $20 \mathrm{~K}, R(T)$ exibits a behavior typical of semimetals or narrow bandgap semiconductors, in line with the previous reports [24, 12]. Below the maximum near $15 \mathrm{~K}$, the resistance becomes metallic-like. The magnetic ordering at $T_{\mathrm{N}}=$ $1.2 \mathrm{~K}$ results in a very abrupt decrease of the resistance in the ordered state.

In external magnetic field (see inset of Fig. 4), both the ordering temperature and the resistance drop below $T_{\mathrm{N}}$ decrease, as expected for an antiferromagnet. These results are in perfect agreement with the DC magnetic susceptibility and specific heat data. The anomalies observed at $1.7 \mathrm{~K}$ in the $\mathrm{AC}$ magnetic susceptibility are not reflected in $R(T)$. Down to $0.4 \mathrm{~K}$, the resistance of our single crystals of ErPdBi remains finite, thus not providing any distinct evidence for superconducting state.

In strong magnetic fields, the resistance of singlecrystalline ErPdBi clearly shows Shubnikov - de Haas

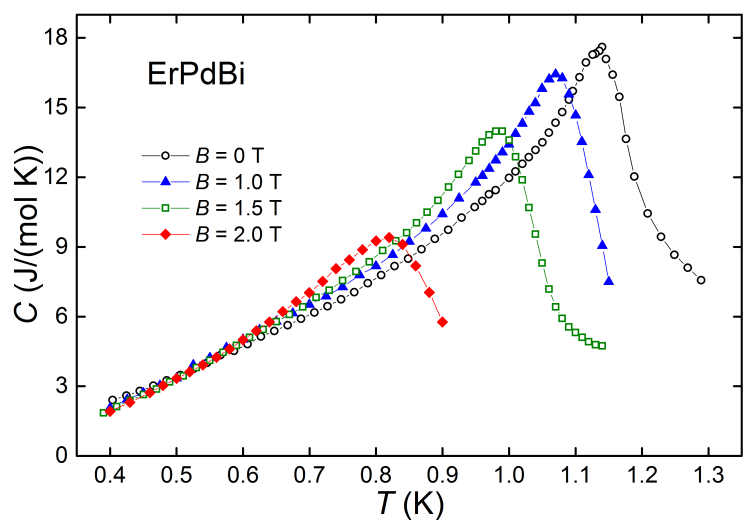

Fig. 3. The specific heat measured below $1.3 \mathrm{~K}$ in several magnetic fields.

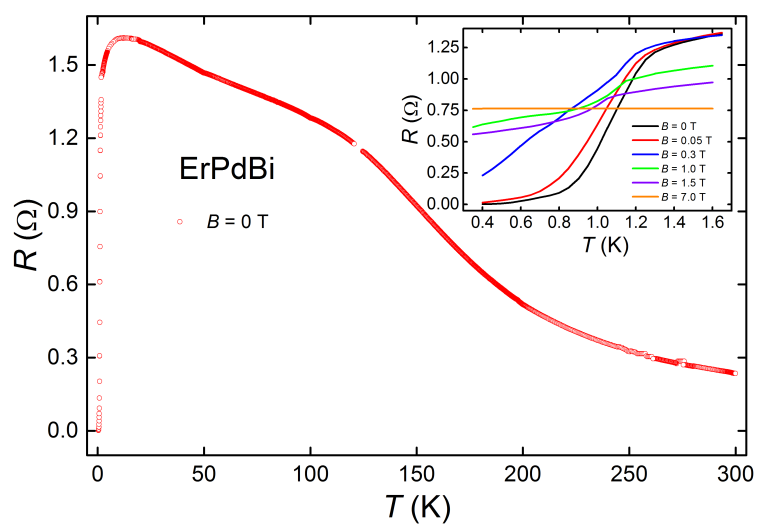

Fig. 4. Electrical resistance as a function of temperature. Inset: Low-temperature resistance in zero and applied magnetic fields.

(SdH) oscillations (Fig. 5), reflecting high mobility of carriers. The $\mathrm{SdH}$ oscillations amplitude decreases with increasing temperature and no oscillations are observed for $T>10 \mathrm{~K}$. A single oscillation frequency $f_{\mathrm{SdH}}=21 \mathrm{~T}$ can be extracted from the fast Fourier transform spectra (inset of Fig. 5). The SdH oscillations frequency is directly

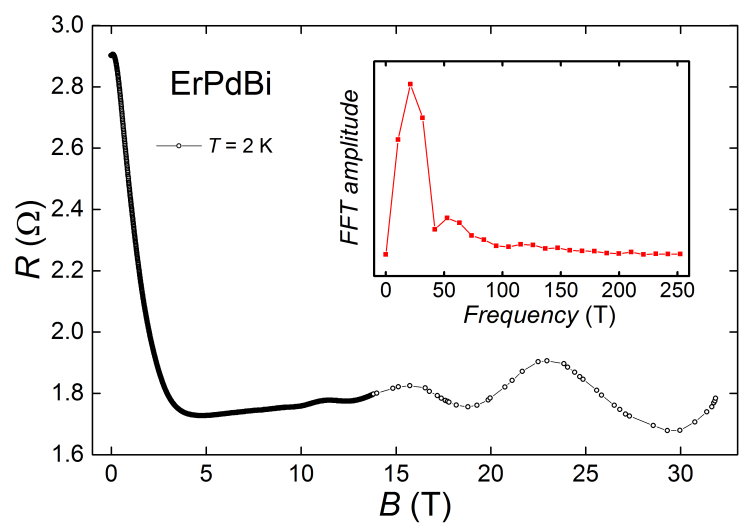

Fig. 5. Field dependence of the resistance at 2 K. Inset: fast Fourier transform (FFT) spectra of SdH oscillations. 


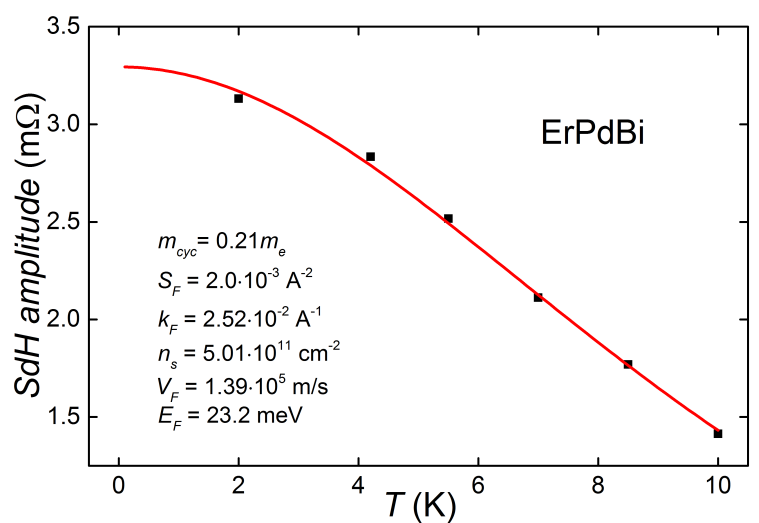

Fig. 6. Temperature dependence of the SdH amplitude. The solid line (red) is best fit to $\lambda(T) / \sinh (\lambda(T))$. A magnetic field of $12.7 \mathrm{~T}$ was used to extract the cyclotron effective mass of $0.21 \mathrm{~m}_{\mathrm{e}}$.

pertained to the cross section $S_{\mathrm{F}}$ of the Fermi surface in momentum space via the Onsager relation: $f_{\mathrm{SdH}}=$ $(\hbar / 2 \pi e) S_{\mathrm{f}}$ where $S_{\mathrm{f}}=\pi k_{\mathrm{F}}^{2}, k_{\mathrm{F}}$ is the Fermi vector, and $e$ stands for the electron charge. Assuming a 2D character of conductivity one can express the 2D surface carrier density as $n_{2 \mathrm{D}}=k_{\mathrm{F}}^{2} / 4 \pi$. By substituting $f_{\mathrm{SdH}}$, the Fermi vector $k_{\mathrm{F}}$ is found to be $0.0252 \AA^{-1}$, that corresponds to the $2 \mathrm{D}$ carrier density of $5.01 \times 10^{-11} \mathrm{~cm}^{-2}$. Furthermore, the temperature dependence of the SdH oscillations amplitude can be fitted to the Lifshitz-Kosevich dependence $\Delta R(T) / \Delta R(0)=\lambda(T) / \sinh (\lambda(T))$, where $\lambda(T)=$ $2 \pi^{2} k_{\mathrm{B}} T m_{\mathrm{cyc}} /(\hbar e B)$, and $m_{\mathrm{cyc}}$ is the cyclotron mass [13]. For ErPdBi this analysis yielded $m_{\mathrm{cyc}}=0.21 m_{\mathrm{e}}$, where $m_{\mathrm{e}}$ is the free electron mass (see Fig. 6); $\Delta R$ was obtained by background subtraction from the resistance measured in the field range $8-32 \mathrm{~T}$. The Fermi level is defined by $E_{\mathrm{F}}=m_{\text {cyc }} V_{\mathrm{F}}^{2}$, where the Fermi velocity $V_{\mathrm{F}}$ is related to $k_{\mathrm{F}}$ by $m_{\mathrm{cyc}} V_{\mathrm{F}}=k_{\mathrm{F}}$. This yielded $E_{\mathrm{F}}=23.2 \mathrm{meV}$ above the Dirac point and $V_{\mathrm{F}}=1.39 \times 10^{5} \mathrm{~m} \mathrm{~s}^{-1}$.

\section{Conclusions}

We prepared high-quality single crystals of the halfHeusler compound ErPdBi and determined its lowtemperature physical properties. At odds with the recent literature report [12], we have not find any clear-cut evidence for the superconducting state below $T_{\mathrm{c}}=1.22 \mathrm{~K}$. Instead, our electrical transport, magnetization and heat capacity studies clearly revealed the antiferromagnetic order at $T_{\mathrm{N}}=1.2 \mathrm{~K}$. Remarkably, this Néel temperature coincides with the reported $T_{\mathrm{c}}$ value and is higher than $T_{\mathrm{N}}=1.06 \mathrm{~K}$ determined in Ref. [12]. Our measurements indicated semimetallic character of the electrical transport in ErPdBi. Observation of the SdH oscillations confirmed the high quality of the single crystals studied. The electronic transport parameters obtained from the $\mathrm{SdH}$ oscillations analysis are similar to those reported in the literature for archetypal topological insulators.

\section{Acknowledgments}

This work was supported by the National Science Centre (Poland) under research grant 2011/01/B/ST3/04466. We are grateful to Prof. V.I. Nizhankovskii for interesting discussion and valuable help in high field measurements.

\section{References}

[1] M.G. Haase, T. Schmidt, C.G. Richter, H. Block, W. Jeitschko, J. Solid State Chem. 168, 18 (2002).

[2] D. Kaczorowski, K. Gofryk, T. Plackowski, A. LeitheJasper, Yu. Grin, J. Magn. Magn. Mater. 290-291, 573 (2005).

[3] K. Gofryk, D. Kaczorowski, T. Plackowski, A. LeitheJasper, Yu. Grin, Phys. Rev. B 72, 094409 (2005).

[4] K. Gofryk, D. Kaczorowski, T. Plackowski, A. LeitheJasper, Yu. Grin, Phys. Rev. B 84, 035208 (2011).

[5] H. Lin, L.A. Wray, Y. Xia, S.Xu, S. Jia, R.J. Cava, A. Bansil, M.Z. Hasan, Nature Mater. 9, 546 (2010).

[6] S. Chadov, X. Qi, J. Kübler, G.G. Fecher, C. Felser, S.C. Zhang, Nature Mater. 9, 541 (2010).

[7] M.Z. Hasan, C.L. Kane, Rev. Mod. Phys. 82, 3045 (2010).

[8] G. Goll, M. Marz, A. Hamann, T. Tomanic, K. Grube, T. Yoshino, T. Takabatake, Physica B 403, 1065 (2008).

[9] N.P. Butch, P. Syers, K. Kirshenbaum, A.P. Hope, J. Paglione, Phys. Rev. B 84, 220504(R) (2011).

[10] T.V. Bay, M. Jackson, C. Paulsen, C. Baines, A. Amato, T. Orvis, M.C. Aronson, Y.K. Huang, A. de Visser, Sol. State Comm. 183, 13 (2014).

[11] F.F. Tafti, T. Fujii, A. Juneau-Fecteau, S.R. de Cotret, N. Doiron-Leyraud, A. Asamitsu, L. Taillefer, Phys. Rev. B 87, 184504 (2013).

[12] Y. Pan, A.M. Nikitin, T.V. Bay, Y.K. Huang, C. Paulsen, B.H. Yan, A. de Visser, Eur. Phys. Lett. 104, 27001 (2013).

[13] D. Shoenberg, Magnetic oscillations in metals, Cambridge Univ. Press, Cambridge 1984. 